\title{
Nutzenbewertung
}

\section{Sie wird zur Bremse für den Nachschub an neuen Arzneimitteln}

\section{In kurzer Zeit ist das zweite neue Arzneimittel an der frühen Nutzenbewertung gescheitert. Die Hersteller wollen es nicht auf den deutschen Markt bringen, weil sie einen schlechten Erstattungsbetrag fürchten.}

Boehringer Ingelheim und Lilly Pharma werden ihren DPP4-Hemmer Linagliptin (Tradjenta ${ }^{\circledR}$ ) zwar in Deutschland in Verkehr bringen, ihn den Patienten aber nicht zur Verfügung stellen. Dem Antidiabetikum drohe ein Erstattungsbetrag, der dem Innovationscharakter nicht angemessen sei, so die Begründung der Unternehmen. Man gehe davon aus, dass die vom Gemeinsamen Bundesausschuss (GBA) getroffene Auswahl der Vergleichstherapie für Linagliptin und die Bewertung zu einer nicht ausreichenden Berücksichtigung des therapeutischen Nutzens und der positiven Eigenschaften des Wirkstoffs führen werden. Die Unternehmen hätten sich gerne einem Vergleich mit den anderen Gliptinen gestellt, sagte eine Sprecherin von Boehringer Ingelheim. Welche Vergleichstherapie der GBA stattdessen festgelegt hat, war allerdings nicht zu erfahren. Darüber hätten Lilly und Boehringer Stillschweigen vereinbart.

\section{Neues Gliptin hierzulande nicht verfügbar}

Das Linagliptin-Präparat wird, da formal weiter im Verkehr, jetzt das komplette Nutzenbewertungsverfahren gemäß dem Arzneimittelmarkt-Neuordnungsgesetz (AMNOG) durchlaufen. Mit der Entscheidung, es Patienten erst gar nicht zur Verfügung zu stellen, verzichteten die Unternehmen zwar auf Umsatz. Gegenüber einer Marktrücknahme erst nach Abschluss des Prozesses habe die gewählte Option aber den Vorteil, dass keine Patienten auf das Produkt eingestellt würden, denen man es später möglicherweise vorenthalten müsse, erklärte die Sprecherin.

Auf jeden Fall lassen Boehringer und Lilly keinen Zweifel daran, dass sie am Ende des Prozesses einen aus ihrer Sicht unangemessen niedrigen Preis nicht akzeptieren würden. Dr. Engelbert Günster, Deutschlandchef von Boehringer, sagt dazu: „Wir können, wenn überhaupt, das neue Antidiabetikum in Deutschland erst zur Verfügung stellen, wenn mehr Transparenz bezüglich des Ausgangs des AMNOGProzesses besteht“. Enttäuscht äußerte er sich auch über die laufenden Gespräche auf Verbandsebene mit den Kostenträgern zur Umsetzung des AMNOG. Diese deuteten darauf hin, „dass zurzeit keinerlei Bereitschaft erkennbar ist, Preise für medizinische Innovationen auch nur angemessen zu gestalten. Im Gegenteil: Es ist die Absicht zu erkennen, die Preise in Deutschland als hoch industrialisiertem Land sogar noch unter dem europäischen Durchschnittspreis festlegen zu wollen“.

Nach einem Bericht der „Frankfurter Allgemeinen Zeitung" konnten sich der GKV-Spitzenverband und die Industrie auch im dritten Anlauf nicht über die Regularien für die anstehenden Preisvereinbarungen für Arzneimittel mit belegtem Zusatznutzen verständigen. Hier dürfte wohl die Schiedsstelle angerufen werden. Linagliptin ist das erste zugelassene Produkt aus der seit Januar 2011 bestehenden Diabetesallianz zwischen Boehringer Ingelheim und Lilly.

\section{Kombi-Blutdrucksenker außer Vertrieb gestellt} Zunächst sah es danach aus, dass es durch das AMNOG entgegen pessimistischen Vorhersagen aus der Pharmabranche nicht zu Verzögerungen der Markteinführung von neuen Arzneimitteln kommen würde. Das Gros der seit dem 1. Januar zugelassenen Präparate ist auch in die Apotheken gekommen, und die Hersteller haben pflichtgemäß ihre Nutzenbewertungsdossiers eingereicht. Zum Schwur kam es aber bereits bei Rasilamlo ${ }^{\circledR}$, der Blutdrucksenker-Fixkombination von Novartis. Der Hersteller hat das Arzneimittel zum 1. September außer Vertrieb gestellt. Streitpunkt war auch hier die vom GBA festgelegte Vergleichstherapie. Es ist damit zu rechnen, dass weitere Unternehmen in vergleichbaren Fällen ebenfalls den Rückzug antreten und einen Bogen um den deutschen Markt machen.

Beim GBA verweist man auf Recht und Gesetz. Der Ausschuss führe die frühe Nutzenbewertung so durch, wie sie vom AMNOG vorgegeben sei. Wenn ein Unternehmen ein Arzneimittel nicht vertreibe, sei dies dessen eigene unternehmerische Entscheidung, sagte ein Sprecher des GBA auf Anfrage.

Bertold Schmitt-Feuerbach
Ausgebremst? In den USA und anderen Ländern Europas nimmt Linagliptin Fahrt auf, in Deutschland bis auf Weiteres nicht.

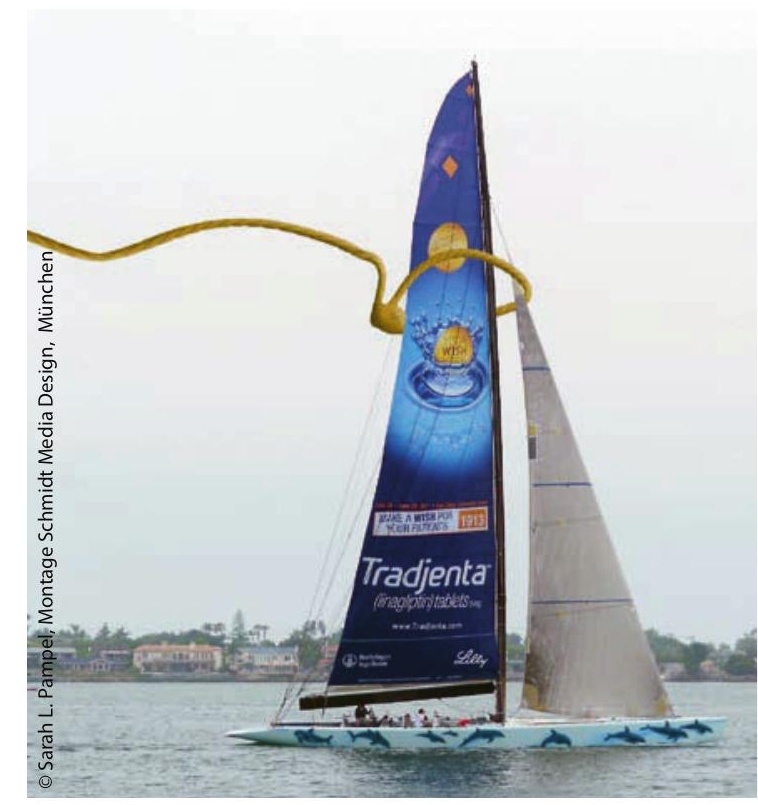

